\title{
论 文
}

\section{FGF 受体-1 和 FGF 受体-2 介导成纤维细胞生长 因子-21 信号传导}

\author{
任桂萍†，尹杰超”，王文飞，李璐，李德山" \\ 东北农业大学生命科学学院, 哈尔滨 150030 \\ $\dagger$ 同等贡献 \\ * 联系人, E-mail: deshanli@163.com \\ 收稿日期: 2009-04-17; 接受日期: 2009-09-14 \\ 黑龙江省科技厅重点攻关项目(批准号: 2006G0461-00)资助
}

\begin{abstract}
摘要成纤维细胞生长因子-21 是 FGF 家族成员, FGF-21 与其他 FGF 家族成员不同, 它没有 促进细胞分裂的作用, 但具有很强的调节血糖作用. 本实验旨在系统研究 FGF-21 的功能受体, 以及配体/受体的作用机制, 为深入研究FGF-21的生物功能, 加快FGF-21 从基因到药物的转变 奠定理论基础. 前期实验表明, FGF-21 促进 3T3-L1 脂肪细胞葡萄糖代谢, 对前脂肪细胞 无作用, 说明 3T3-L1 脂肪细胞表达 FGF-21 功能受体. 本文以 3T3-L1 脂肪细胞为靶标, 深入研究 FGF-21 的功能受体. 在 FGF-21 作用下 3T3-L1 脂肪细胞中的 FGF 受体 1(R1)和 FGF 受体 2(R2)形成特异性异源二聚体. 脂肪细胞经 FGF-21 处理后, R1 和 R2 均迅速发生磷酸 化，表明两个受体均被 FGF-21 激活. siRNA 干涉实验表明，抑制 R1 和 R2 功能可以抑制 FGF-21 处理后 3T3-L1 脂肪细胞 Glut1 的表达水平, 揭示了 FGF-21 通过 R1 和 R2 上调 3T3-L1 脂肪 细胞中 Glut1 的表达. 免疫印迹结果表明, FGF-21 可以介导 3T3-L1 脂肪细胞表面 R1 和 R2 的内吞. 克隆后测序分析结果表明, 3T3-L1 脂肪细胞表达的 FGF 受体为 FGFR-1IIIc 和 FGFR-2IIIc. 结果表明, FGFR-1IIIc 和 FGFR-2IIIc 是 FGF-21 的功能受体, 参与 FGF-21 在脂肪细胞中介导的糖代谢活性的信号传导.
\end{abstract}

\section{关键词}

FGF-21

FGF 受体-1

FGF 受体-2

葡萄糖代谢

Glut1
成纤维细胞生长因子 (fibroblast growth factor, FGF) 在机体内的各种生物活动中起重要作用, 如细 胞调亡、细胞生存、细胞趋向性、细胞黏附、细胞迁 移、细胞分化和细胞增殖等 ${ }^{[1,2]}$. 迄今为止, 已经报道 了 23 个 FGF 成员 ${ }^{[2,3]}$. FGF 有 4 种酪氨酸蛋白激酶受 体 (fibroblast growth factor receptor, FGFR), 即 FGFR-1 FGFR-4. 虽然存在 FGFR-5, 但由于缺少细
胞内部分 ${ }^{[4]}$, 因此无受体活性. FGFR 属于 Ig 超家族, 与该家族的其他成员一样, 它的结构包括 $\mathrm{N}$ 端信号 肽, Ig I, Ig II, IgIII, 在 Ig I 和 Ig II 之间有一个酸盒, 跨膜区和胞内的酪氨酸激酶区域 ${ }^{[1,2]}$. Ig II 和 IgIII及其 之间的连接序列决定了受体结合配体的特异性 ${ }^{[4,5]}$. 相反, Ig I 及 Ig I 和 Ig II 之间的连接序列对受体的亲 和力起相反作用 ${ }^{[5]}$. 除 FGFR-4 外, 所有的跨膜 FGFR

英文版见: Ren G P, Yin J C, Wang W F, et al. Fibroblast growth factor (FGF)-21 signals through both FGF receptor-1 and 2. Sci China Life Sci, 2010, doi: 10.1007/s11427-010-0086-4 
均有两种 IgIII剪切异构体, 命名为 IgIIIb 和 IgIIIc, 两 种异构体具有相同的 $5^{\prime}$ 端序列，但 $3^{\prime}$ 端序列则由不同 剪切形成, 这决定了受体结合配体的特异性. 因此, 共有 7 个 FGFR 剪切异构体 ${ }^{[1]}$. 此外, 其他部位的突 变, 如缺少 Ig I, 跨膜区氨基酸的改变等都可以改变 受体结合配体的特异性和生物反应效果.

典型的 FGF/受体复合物由 2 个配体和 2 个受体及 肝素样氨基葡聚糖 (heparan-like glycosaminoglycans, HLGAGs)组成, HLGAGs 的作用是稳定 $\mathrm{FGF} /$ 受体复 合物的结构 ${ }^{[6-9]}$. 然而, FGF 与其受体的结合是一个 复杂的过程. 一种 FGF 可以分别与多种 FGFR 结合, 如 FGF-1 可与所有 4 种受体及其剪切异构体反应 ${ }^{[1]}$. 可是有些 FGF 只与一种受体反应，如 FGF-19 特异性 地与 FGFR-4 反应 ${ }^{[10]}$. 另一方面, 一种受体可与多种 配体反应, 取决于反应的组织和细胞内外环境, 但是 根据不同的配体和所招募的针对特异性受体针定位点 的不同信号蛋白使受体激活, 从而发出不同信号 ${ }^{[1,11]}$.

FGF 家族成员曾被公认为是细胞增殖、生存以及 血管形成和细胞分化的促进剂. 但近年来新发表的资 料表明, FGF 家族中的一些成员在体内代谢过程中起 非常重要的作用, 如脂代谢和糖代谢 ${ }^{[10,12 \sim 14]}$. FGF-21 可促进鼠 3T3-L1 脂肪细胞和人脂肪细胞吸收葡萄 糖 ${ }^{[15,16]} . \mathrm{ob} / \mathrm{ob}$ 和 $\mathrm{db} / \mathrm{db}$ 小鼠实验表明, FGF-21 不仅降 低血糖, 还可以降低血液中的甘油三酯. 更令人感兴 趣的是, 与其他成员不同, FGF-21 没有促进细胞分裂 的作用, 不结合肝素, 其生物活性不依赖于肝素的存

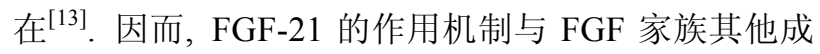
员不同.

为了研究 FGF-21 的作用机制, 本实验明确了 FGF-21 的功能受体. 本实验室已经报道了 FGF-21 与 $3 \mathrm{~T} 3-\mathrm{L} 1$ 脂肪细胞的膜表面蛋白可以形成 2 个复合物 ${ }^{[17]}$, 在 FGF-21/受体复合物中检测到 FGFR-2 的存在 ${ }^{[15]}$. FGF-21 可使 FGFR-1 和 FGFR-2 磷酸化 ${ }^{[13]}$. 本文进一 步研究了 FGF-21 与 FGFR-1 和 FGFR-2 在物理、化 学和生物学上的深层次关系.

\section{1 材料与方法}

\section{1 细胞培养}

(1) 3T3-L1 脂肪细胞的分化. 鼠 3T3-L1 细胞购自 ATCC(American Tissue Culture Collections), 用含 10\%
小牛血清和 1\%抗生素(Invitrogen, Carlsbad Carlsbad, CA)的 DMEM 培养基培养和维持 3T3-L1 成纤维细胞 (即前脂肪细胞). 将生长状态良好的 3T3-L1 成纤维 细胞用含 $10 \%$ 胎牛血清, $1 \%$ 抗生素, $5 \mu \mathrm{g} / \mathrm{mL}$ 胰岛素 (Sigma, St. Louis, MO), $0.25 \mu \mathrm{mol} / \mathrm{L}$ 地塞米松(Dex, Sigma)和 $0.5 \mathrm{mmol} / \mathrm{L} 3$-异丁基-1-甲基黄嘌呤(IBMX, Dex, Sigma)的脂肪细胞分化液 1 培养 2 天. 再用含 $10 \%$ 胎牛血清, $1 \%$ 抗生素和 $5 \mu \mathrm{g} / \mathrm{mL}$ 胰岛素的脂肪细 胞分化液 2 培养 2 天. 然后用含 $10 \%$ 胎牛血清和 $1 \%$ 抗生素的 DMEM 培养基培养直至细胞分化成脂肪细 胞备用.

(2) BaF-3 细胞培养. BaF-3 细胞的生长、繁殖 依赖于 IL-3 的存在. 用含 $10 \%$ 胎牛血清, $1 \%$ 抗生素和 $5 \mathrm{ng} / \mathrm{mL}$ IL-3(R\&D systems, Inc. Minneapolis, MN)的 1640 培养基培养 $\mathrm{BaF}-3$ 细胞.

\subsection{FGF-21 生物素化}

纯化 FGF-21 蛋白方法见文献[15 17]. 用葡萄糖 吸收实验检测其生物活性, 有活性的用于下一步实 验. 按照生物素标记试剂盒(Cat. No: 21336, Pierce Biotechnology Inc. Rockford, IL)推荐的方法使 FGF21 生物素化. 具体方法: FGF-21 与 50 倍的 biotin-NHS 混合, 混合物冰浴 $2 \mathrm{~h}$, 用色谱方法去除未标记 biotinNHS. 通过 3T3-L1 脂肪细胞的葡萄糖吸收实验检测 生物素化的 FGF-21 生物活性, 只有保持可促进葡萄 糖吸收功能的 FGF-21 蛋白才能用于本研究.

\section{3 偶联实验}

在 $37^{\circ} \mathrm{C}$ 下, 3T3-L1 脂肪细胞无血清饥饿 $2 \mathrm{~h}$ 后, 加入生物素化的 FGF-21, 在 $4^{\circ} \mathrm{C}$ 与脂肪细胞单层细 胞捊育 $2 \mathrm{~h}$. 用预冷的 Hanks 缓冲液清洗细胞, 除去 游离的配体. 然后, 加入非还原性偶联剂 $\mathrm{BS}^{3}(\# 21580$, Pierce), 在 $4^{\circ} \mathrm{C}$ 与脂肪细胞单层孵育 $2 \mathrm{~h}$. 除去偶联剂 后, 加入含 $50 \mathrm{mmol} / \mathrm{L}$ Tris, $150 \mathrm{mmol} / \mathrm{L} \mathrm{NaCl}, \mathrm{pH} 7.5$ 的缓冲液, $37^{\circ} \mathrm{C}$ 作用 $15 \mathrm{~min}$ 终止反应. 用裂解液(含 $50 \mathrm{mmol} / \mathrm{L}$ Tris, $1 \%$ triton $\mathrm{X}-100,0.1 \%$ chaps, 150 $\mathrm{mmol} / \mathrm{L} \mathrm{NaCl}, 1 \mathrm{mmol} / \mathrm{L} \mathrm{EDTA}$ )和蛋白酶抑制剂混合 物(Roche, Indianapolis, IN) 裂解细胞. 裂解物脱脂上 清和沉淀用于免疫沉淀. 由于使用的配体是生物素 化的 FGF-21, 用链霉亲和素琼脂糖(Sigma)免疫沉淀 (immunoprecipitation, IP)FGF-21/受体复合物, IP 产物 在 SDS-PAGE 电泳中分离后, 用 FGF-21 抗体或 FGFR 
抗体进行免疫印迹检测 FGF-21/受体复合物.

\section{4 免疫印迹}

兔抗 FGFR-2 多抗购自 Santa Cruz 公司(sc-122, Santa Cruz Biotechnology, Inc., Santa Cruz, CA). 以 FGFR-1 和 FGFR-3 的 C 端氨基酸序列为抗原分别制 备兔抗 FGFR-1 多抗和兔抗 FGFR-3 多抗. 为验证这 些抗体的特异性, 分别将鼠 FGFR-1 FGFR-3 克隆到 逆转录病毒载体上, 然后稳定转染不表达 FGFR 的 BaF-3 细胞. 用含 $0.1 \mu \mathrm{g} / \mathrm{mL}$ FGF-1 培养基篎选表达 FGFR 的 BaF-3 细胞. 以表达 FGFR 细胞的裂解液为材 料, 用这些抗体进行免疫印迹来验证兔抗鼠 FGFR-1 FGFR-3 抗体的特异性. 制备 FGFR-1 抗体的多肽序 列: RHPTQLANSGLKRR. 制备 FGFR-3 抗体的多肽 序列：LLPPGPPSNGGPRT. 这些多肽序列由金思特 公司合成.

在还原条件下，将细胞裂解物或免疫沉淀物沸 水浴 $8 \mathrm{~min}$, 样品在 Tris-Glycine 蛋白胶上分离. 样品 分离后转移到硝酸纤维素膜(Invitrogen)上, 用 $5 \%$ 的 脱脂牛奶封闭硝酸纤维素膜, 然后与特异性抗体杂 交, 用相应的标有 HRP 的二抗室温孵育, 用 SuperSignal(Pierce Biotechnology Inc., Rockford, IL)检测试 剂检测反应信号.

\subsection{T3-L1 脂肪细胞中 FGFR 的分析}

(1) 3T3-L1 脂肪细胞中 FGFR 磷酸化分析. 分化 14 天的 3T3-L1 成纤维细胞无血清饥饿 $2 \mathrm{~h}$ 或 3T3-L1 脂肪细胞无血清饥饿过夜, $37^{\circ} \mathrm{C}, 1 \mu \mathrm{g} / \mathrm{mL}$ FGF-21 或 $0.1 \mu \mathrm{g} / \mathrm{mL}$ FGF-1 处理 $10 \mathrm{~min}$, 立即用含磷酸酶抑制 剂(Sigma)的裂解缓冲液裂解细胞，脱脂、去沉淀的裂 解液用于免疫沉淀.

用抗磷酸化酪氨酸抗体(4G10, Upstate)免疫沉淀 磷酸化酪氨酸蛋白, 用 FGFR 抗体免疫印迹, 或反之 用 FGFR 抗体免疫沉淀, 用抗磷酸化酪氨酸抗体免疫 印迹.

（2）配体介导的受体降解作用. 分化 18 天的 3T3-L1 脂肪细胞无血清饥饿过夜, 用 $5 \mu \mathrm{g} / \mathrm{mL}$ FGF21 或 $0.1 \mu \mathrm{g} / \mathrm{mL}$ FGF-1 处理 $24 \mathrm{~h}$. 用含有蛋白酶抑制 剂 (Roche) 的裂解缓冲液裂解细胞, 通过 $8 \%$ SDSPAGE 电泳分析裂解物. 用特异性的受体抗体通过免 疫印迹检测由配体介导的受体降解, 从而造成受体 表达量的减少, 以 $\beta$-actin 作为内参. 重组人 FGF-1 购
自 R\&D 公司(232-FA).

(3) 构建逆转录病毒载体和转染 BaF-3 细胞. 将 FGFR cDNA 克隆到 $3^{\prime}$ 末端带有 flag 标签(DYDDDK) 序列的复制缺陷的逆转录病毒表达载体中. 此载体 以鼠白血病病毒为骨架构建, 并含有 CMV 启动子. 此外, 转录元件包含一个核糖体插入位点, 此位点可 以促进新霉素磷酸转移酶的翻译, 而新震素磷酸转 移酶可以利用新需素篎选转化子. 为了获得能稳定表 达 FGFR 的 BaF-3 细胞, 用 FuGene(Roche)转染试剂 将携带 FGFR 基因的逆转录病毒表达载体转染包装 细胞 EcoPack(Mountain View, CA); 包装细胞上清液 感染 BaF-3 细胞; 用含 $0.1 \mu \mathrm{g} / \mathrm{mL} \mathrm{FGF}-1$ 和 $10 \mu \mathrm{g} / \mathrm{mL}$ 肝素，而无 IL-3 的培养基篮选稳定表达 FGFR 的 BaF-3 细胞; 所获得的阳性细胞克隆用于 FGFR 分析.

(4) RNA 干涉实验. FGFR-1 和 FGFR-2 的小干 涉 RNA 二聚体来自于 Ambion 公司(Ambion, Inc., Austin, TX)的预设计库. FGFR-1(NMOL/L_010206)基 因特异性的小干涉 RNA 是 21 个核苷酸的二聚体，正 义链为 $\mathrm{S} 1$, 反义链为 $\mathrm{A} 1$ (ID\#66823). FGFR-2(NMOL/L 201601)基因特异性的小干涉 RNA 是 21 个核苷酸的 二聚体, 正义链为 $\mathrm{S} 2$, 反义链为 $\mathrm{A} 2(\mathrm{ID} \#$ 66824). 与 哺乳动物序列无同源性的不规则 siRNA(Ambion)作 为阴性对照. 用胰蛋白酶消化分化 7 天的 3T3-L1 脂 肪细胞, 用电穿孔系统(Bio-Rad Laboratories, Hercules, CA) 将 $5 \mathrm{nmol} / \mathrm{L}$ siRNA(FGFR-1 特异性的, FGFR-2 特 异性的或 FGFR-1 和 FGFR-2 联合体的)电转化密度为 $1 \times 10^{5}$ 消化后的细胞. 电转化的细胞重新接种至 6 孔 板中, $37^{\circ} \mathrm{C}$ 下培养 2 天. 为了检测 FGFR-1 和 FGFR-2 在 FGF-21 诱导的 Glut1 上调表达中的作用, siRNA 感 染细胞无血清饥饿处理 $3 \mathrm{~h}$, 然后用 $0.1 \mu \mathrm{g} / \mathrm{mL}$ FGF-21 处理 $4 \mathrm{~h}$. 提取处理细胞的总 RNA，用实时苂 光定量 PCR 检测处理后的细胞中 FGFR-1, FGFR-2 和 Glut1 的表达水平.

FGFR-1 siRNA: S1: 5'-GGAGAAAGAGACGGA CAACTT-3'; A1: 5'-GUUGUCCGUCUCUUUCUCCT C-3'; FGFR-2 siRNA: S2: 5'-GGAUACCACUUUAGA ACCATT-3'; A2: 5'-UGGUUCUAAAGUGGUAUCCT C-3'.

(5) 实时荧光定量 PCR. 用 Trizol 试剂提取 siRNA 感染后的 3T3-L1 细胞总 RNA. 在 SuperScript ${ }^{\mathrm{TM}} \mathrm{III}$ 反 转录酶(Invitrogen)作用下, $2 \mu \mathrm{g}$ 总 RNA 进行 RT-PCR 反应. 实时荧光定量 PCR 在实时荧光定量 PCR 仪 
(ABI 7500)中完成. FGFR-1(ID number: MM 00438923_m1), FGFR-2(ID number: MM 00438941_ $\mathrm{m} 1$ )和 rRNA(ID number: 4310893E)的引物及探针来自 于 $\mathrm{ABI}$ 库. 用 Primer Express 软件设计 Glut-1 的引物 和探针, 以 $18 \mathrm{~S}$ rRNA 作为内参.

Glut1 的正向引物 (F1) 和反向引物 (R1): F1: 5'-GCCCCCAGAAGGTTATTGA-3'; R1: 5'-CGTGGT GAGTGTGGTGGATG-3'; 探针序列: 5'-TTCTACAA TCAAACATGGAACCACCGCA-3'.

\section{6 克隆和序列分析鼠 FGFR 剪切异构体}

利用高保真酶(TaKaRa)从 3T3-L1 细胞中克隆鼠 FGFR 及其剪切异构体基因, 所用引物设计于 $5^{\prime}$ 和 $3^{\prime}$ 非翻译区. FGFR-1 的正向引物和反向引物分别命名 为 $\mathrm{F} 2$ 和 $\mathrm{R} 2, \mathrm{FGFR}-2$ 的正向引物和反向引物分别命名 为 $\mathrm{F} 3$ 和 R3. PCR 扩增产物克隆至 $\mathrm{pMD} 18-\mathrm{T}$ 载体上 (TaKaRa). 对于每种受体, 随机挑选 100 个单菌落,

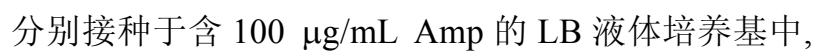
$37^{\circ} \mathrm{C}$ 过夜培养, 提取质粒方法按 Qiagen 公司质粒小 提试剂盒说明. 为鉴定每种受体的剪切异构体, 所有 克隆都进行测序和生物分析. 引物序列: F2: 5'-CAG AACTGGGATGTGGGGCTGGAA-3'; R2: 5'-GGGAG AAAACTGGGGACAGGGTTGGTAG-3'; F3: 5'-GCC CATGGTCTCAGAAGAAGTG-3'; R3: 5'-GTTCCTG GTGCTGTCCTGTTTG3'.

\section{2 结果}

\subsection{FGF-21/受体复合物含有 FGFR-1 和 FGFR-2}

FGF-21 可促进 3T3-L1 脂肪细胞对葡萄糖的吸收 作用，但对未分化的 3T3-L1 细胞却没有作用 ${ }^{[13,15 \sim 17]}$, 因此推断分化后的 3T3-L1 脂肪细胞表达 FGF-21 的 功能受体. 为了寻找 FGF-21 在脂肪细胞中的功能受 体, 将生物素化的 FGF-21 偶联至细胞表面, 在大约 $300 \sim 400 \mathrm{kD}$ 分子量之间出现了 2 条 FGF-21/受体复合 物条带 ${ }^{[15,17]}$. 只有脂肪细胞能检测到复合物, 而前脂 肪细胞没有, 这与葡萄糖吸收实验结果相一致. 这说 明所形成的配体/受体复合物是 FGF-21 特异性的 ${ }^{[15]}$. 由于 FGF-21 是 FGF 家族成员, 因此用各种 FGFR 抗 体对复合物进行免疫检测. 以人 FGFR-2 为抗原制备 的商品化 FGFR-2 抗体能与鼠源 FGFR-2 发生反应. 据此, 以鼠 FGFR-1 和 FGFR-3 的 C 端多肽为抗原制
备兔抗鼠 FGFR-1 和 FGFR-3 抗体(见材料与方法). 由 于 3T3-L1 成纤维细胞和 3T3-L1 脂肪细胞均不表达 FGFR-4, 因此没有制备 FGFR-4 抗体(结果未显示). 为了鉴定抗体的特异性，将携带鼠 FGFR-1 FGFR-3 基因的逆转录病毒载体转染至不表达 FGFR 的 BaF-3 细胞中. 图 1 表明, 这些抗体特异性地识别相应的受 体，不同受体之间无交叉反应. 同时，用 FGFR-1 抗 体通过免疫印迹发现 2 条复合物条带都含有 FGFR-1. 除了含有 FGFR-1 外, 用 FGFR-2 抗体通过免疫印迹 发现分子量较大的复合物还含有 FGFR-2. FGFR-3 抗 体不能识别这 2 个复合物, 说明在脂肪细胞中 FGFR3 与 FGF-21 无关. 根据上述结果, 将研究定位在 FGFR-1 和 FGFR-2(图 2).

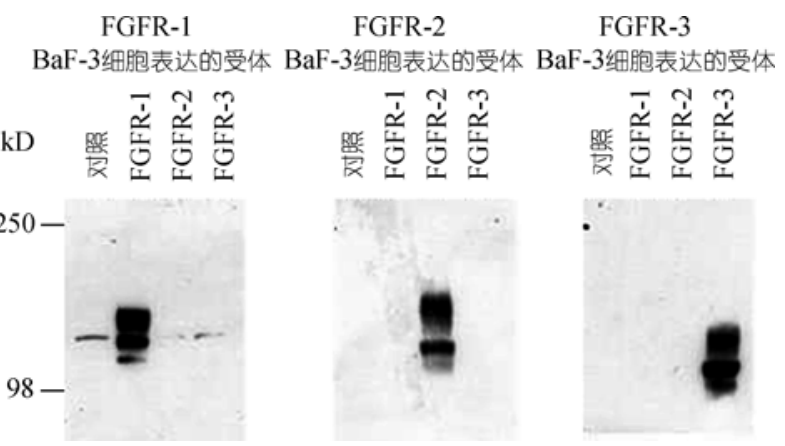

图 1 鉴定兔抗鼠 FGFR-1 FGFR-3 抗体特异性 对照: 不表达受体的 BaF-3 细胞裂解样品; FGFR-1: 表达 FGFR-1 的 BaF-3 细胞裂解样品; FGFR-2: 表达 FGFR-2 的 BaF-3 细胞裂解 样品; FGFR-3: 表达 FGFR-3 的 BaF-3 细胞裂解样品

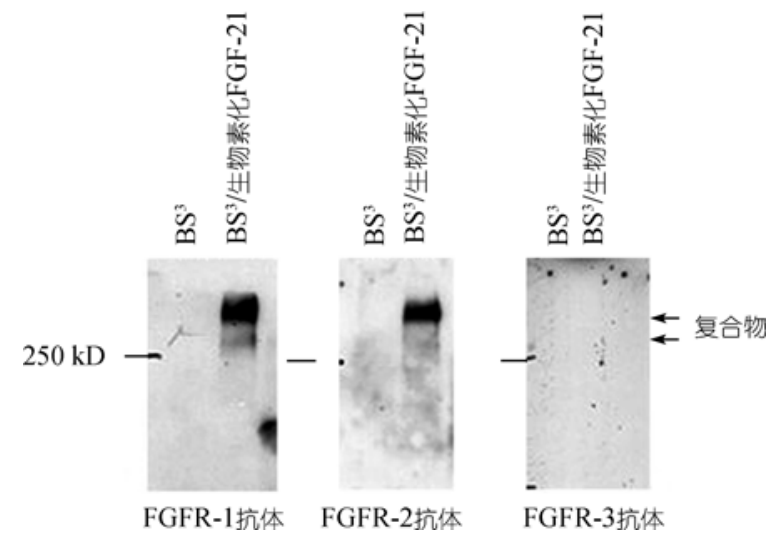

图 2 鉴定 FGF-21/受体复合物中的 FGFR

$\mathrm{BS}^{3}$ : 未经生物素化的 FGF-21 处理, 只加 $\mathrm{BS}^{3}$ 偶联剂的 3T3-L1 脂 肪细胞裂解样品; $\mathrm{BS}^{3}$ /生物素化 FGF-21: 经生物素化的 FGF-21 处 理后, 加 $\mathrm{BS}^{3}$ 偶联剂的 3T3-L1 脂肪细胞裂解样品 
2.2 FGF-21 诱导 3T3-L1 脂肪细胞中 FGFR-1 和 FGFR-2 磷酸化

若 FGFR-1 和 FGFR-2 是 FGF-21 的功能受体, 则 推测 FGF-21 像 FGF 家族其他成员一样能使其受体磷 酸化 ${ }^{[1]}$. 用抗磷酸化酪氨酸抗体进行 IP, 受体抗体进 行免疫印迹检测, 发现 FGF-21 处理后, 这 2 种受体 都发生了磷酸化(图 3(A)). 为证实结果的可信性, 进 行了反向 IP, 即用受体抗体进行 IP, 用抗磷酸化酪氨 酸抗体进行免疫印迹, 得到同样结果(图 3(B)). 与偶 联结果一致，相同剂量的 FGF-21 不能使 3T3-L1 前脂 肪细胞中的 FGFR-1 和 FGFR-2 磷酸化(图 3(C)). 与 预期结果一致, 阳性对照 FGF-1 均可使 3T3-L1 前脂 肪细胞中这 2 种受体磷酸化. 这些结果与 FGF-21 可 促进 3T3-L1 脂肪细胞对葡萄糖的吸收作用一致, 即 FGF-21 处理只能使脂肪细胞中的这 2 种受体磷酸化, 对前脂肪细胞中的这 2 种受体无作用.

\subsection{FGF-21 引起 BaF-3 细胞表达的 FGFR-2 磷酸化}

为了证实 FGF-21 与其受体的特异性反应, 从 3T3-L1 脂肪细胞中克隆了 FGFR-2 全长 cDNA, 并将 其构建在逆转录病毒载体上, 转染 BaF-3 细胞, 用含 $0.1 \mu \mathrm{g} / \mathrm{mL}$ FGF-1 和 $10 \mu \mathrm{g} / \mathrm{mL}$ 肝素的培养基篮选阳性 细胞. 表达 FGFR-2 的阳性细胞用于检测 FGF-21 与 其受体的特异性反应. 表达 FGFR-2 的 BaF-3 细胞无 血清饥饿过夜; 不加肝素, 在 $37^{\circ} \mathrm{C}$ 下, 用 FGF-21 处 理饥饿细胞 $10 \mathrm{~min}$. 抗磷酸化酪氨酸抗体免疫沉淀 磷酸化 FGFR-2, 用 FGFR-2 抗体进行免疫印迹. FGF-21 引起 FGFR-2 磷酸化作用呈剂量依赖性, 在 FGF-21 剂量较高时磷酸化效果明显(图 4). 肝素对 FGF-21 引起的 FGFR 磷酸化作用几乎没有影响(结果 未显示), 与相关报道一致 ${ }^{[13]}$.

\subsection{FGF-21 介导 3T3-L1 脂肪细胞中 FGFR-1 和 FGFR-2 的内吞}

许多生长因子的配体/受体复合物内吞后, 最终 被细胞内的酶降解; 若 FGFR-1 和 FGFR-2 是 FGF-21 的受体, FGF-21 即可介导 FGFR-1 和 FGFR-2 的内吞 及降解. 为了证实这个假设, FGF-21 处理分化 18 天 的 3T3-L1 脂肪细胞 $24 \mathrm{~h}$, 用受体特异性的抗体进行 免疫印迹检测 FGFR-1 和 FGFR-2. 图 5 表明，与未处 理的细胞相比较, 经过 FGF-21 处理的 3T3-L1 脂肪细 胞中 FGFR-1 和 FGFR-2 的表达量明显降低, 无论是
成熟蛋白还是其前体都受到了影响. 因此, 上述结果 证实了本实验假设, 即 FGFR-1 和 FGFR-2 介导 FGF21 信号传导.

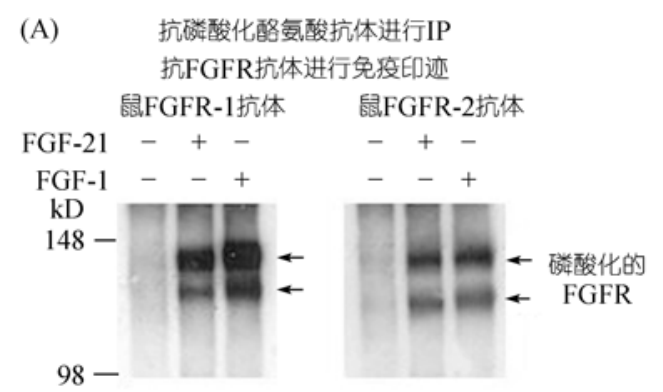

(B)

$$
\begin{gathered}
\text { 抗FGFR抗体进行IP } \\
\text { 抗磷酸化酪氨酸抗体进行免疫印迹 }
\end{gathered}
$$

鼠FGFR-1抗体鼠FGFR-2抗体

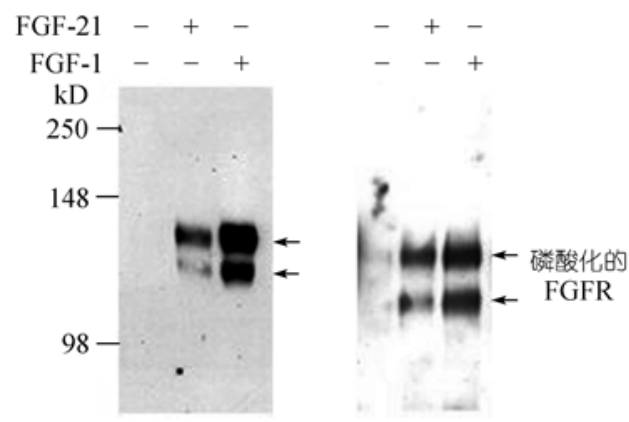

用进行IP的抗体再进行免疫印迹检测

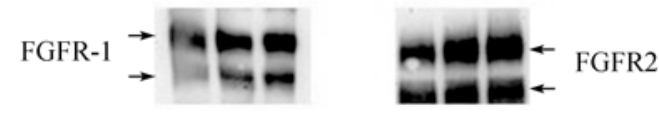

(C) 抗磷酸化酪氨酸抗体进行IP 抗FGFR抗体进行免疫印迹 抗FGFR-1抗体抗FGFR-2抗体

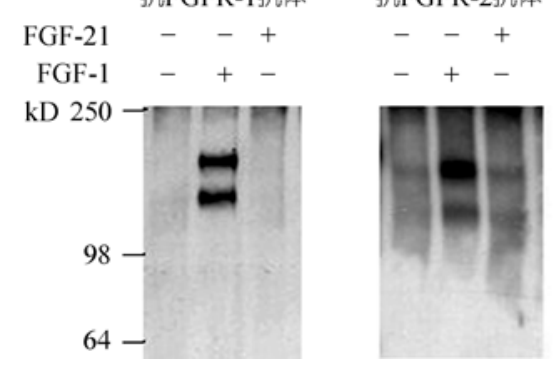

图 3 FGF-21 诱导 3T3-L1 脂肪细胞和成纤维细胞中 FGFR-1 和 FGFR-2 磷酸化作用

(A) 用抗磷酸化酪氨酸抗体进行 IP, FGFR 抗体进行免疫印迹检测 FGFR 磷酸化情况; (B) 用 FGFR 抗体进行 IP, 抗磷酸化酪氨酸抗 体进行免疫印迹检测 FGFR 磷酸化情况. 为使操作标准化, 用进 行 IP 反应的相应 FGFR 抗体再次进行免疫印迹, 从而去除背景干 扰; (C) 用抗磷酸化酪氨酸抗体进行 IP, FGFR 抗体进行免疫印迹检 测 FGFR 磷酸化情况 


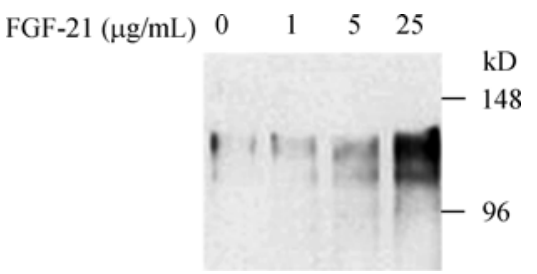

图 4 FGF-21 引起 BaF-3 细胞表达的 FGFR-2 磷酸化作用 表达 FGFR-2 的 BaF-3 细胞分别用不同浓度的 FGF-21 处理, 立即 用含酪氨酸磷酸酶抑制剂的裂解缓冲液裂解细胞, 抗磷酸化酪氨 酸抗体免疫沉淀磷酸化 FGFR-2, 用 FGFR-2 抗体进行免疫印迹

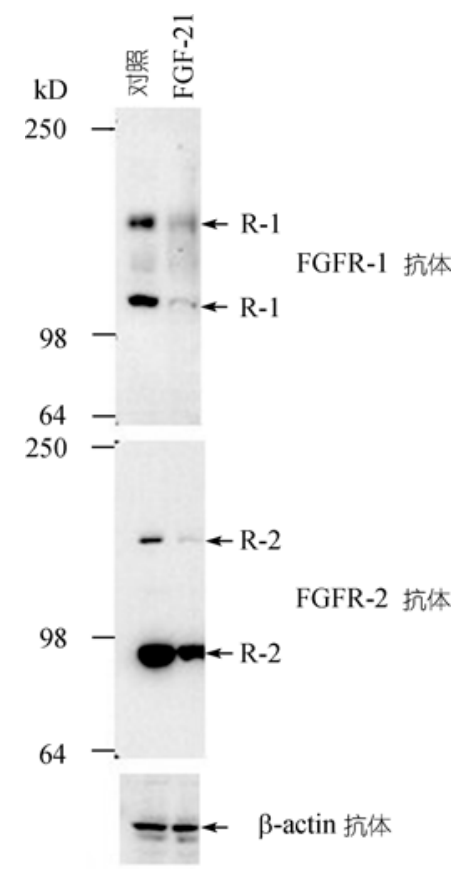

图 5 FGF-21 介导受体降解

对照: 未经处理的 3T3-L1 脂肪细胞; FGF-21: FGF-21 处理的 3T3-L1 脂肪细胞, 以 $\beta$-actin 作为内参

\subsection{FGFR-1 和 FGFR-2 特异性 siRNA 可消除 FGF-21 诱导的 Glut1 上调}

为检测 FGFR-1 和 FGFR-2 的表达在 FGF-21 诱 导的 Glut1 上调中的作用, 设计了 FGFR-1 和 FGFR-2 特异性的 siRNA, 并电转化 3T3-L1 脂肪细胞, 从而 抑制FGFR-1 和 FGFR-2 基因表达. 电转化 2 天后，提 取细胞的 RNA，利用实时荧光定量 PCR 检测 FGFR-1 和 FGFR-2 表达水平. 图 6(A)和(B)表明，基因特异性 的 siRNA 可抑制 FGFR-1 和 FGFR-2 的表达, 不规则 siRNA 对这 2 种受体都没有作用. 为了检测抑制 FGFR 对 FGF-21 诱导 Glut1 上调的影响, $0.1 \mu \mathrm{g} / \mathrm{mL}$ FGF-21
处理 siRNA 转染细胞 $4 \mathrm{~h}$, 利用实时苂光定量 PCR 检 测 Glut1 表达水平. 图 6(C)结果表明, FGF-21 能显著 上调不规则 siRNA 转染细胞中的 Glut1 表达水平, 但 是这种上调作用可以完全被 FGFR-1 特异性的 siRNA, FGFR-2 特异性的 siRNA 或 FGFR-1 和 FGFR-2 联合 体的 siRNA 消除, 这种结果可能是由于 FGFR-1 和 FGFR-2 表达量减少而产生. 上述结果表明, FGFR-1 和 FGFR-2 介导 FGF-21 诱导的 Glut1 上调.

\subsection{T3-L1 细胞中表达的 FGFR 剪切异构体是 FGFR-1IIIc 和 FGFR-2IIIc}

本研究表明, FGFR-1 和 FGFR-2 调控 FGF-21 促 进葡萄糖吸收. 但是参与反应的究竟是受体的哪种 剪切异构体呢? 根据 $5^{\prime}$ 和 3 '端非翻译区设计特异性 引物，利用 RT-PCR 技术扩增 FGFR-1 和 FGFR-2 的 cDNA, 并克隆至 pMD18-T 载体上. 每个受体都随机 挑取 100 个阳性克隆进行序列分析. 测序结果表明, 尽管存在一些突变, 但是这 2 种受体的所有阳性克隆 都是 IgIIIc 剪切异构体. 所产生的突变包括 IgI 的缺 失和单个氨基酸的置换. 图 7 是随机挑取的阳性克隆 测序结果，其序列分别与 NCBI 上已发表的 FGFR-1 和 FGFR-2 的 IgIIIc 剪切异构体一致.

\section{3 讨论}

FGF-21 可促进 3T3-L1 脂肪细胞对葡萄糖的吸 收作用 ${ }^{[13,15 \sim 17]}$, 但对未分化的 3T3-L1 前脂肪细胞(成 纤维细胞)却没作用. 因此推断, 3T3-L1 脂肪细胞中 表达 FGF-21 的功能受体, 3T3-L1 脂肪细胞可作为细 胞模型研究 FGF-21 的功能受体. 利用偶联技术, FGF-21 特异性地与分化的 3T3-L1 脂肪细胞表面的受 体形成复合物, 并呈剂量依赖性. 已知在相同条件下, 未分化的 3T3-L1 成纤维细胞中 FGF-21 不能形成任 何复合物，上述结果表明，这些假设的受体是脂肪细 胞特异性的，这与葡萄糖吸收实验结果一致. 复合物 的分子量大约在 300 400 kD, 这符合 FGF 家族成员 形成配体/受体复合物的规律, 即每个复合物中含有 2 个受体和 2 个配体 ${ }^{[6,7]}$. 已发表数据表明, FGF 家族 成员形成配体/受体复合物中有 4 种受体，即 FGFR-1 FGFR-4. 为了研究复合物组成, 用特异性的 FGFR 抗 体进行免疫印迹检测复合物. 本研究发现, 复合物中 同时含有 FGFR-1 和 FGFR-2. 本研究检测了这 2 种 
受体对 FGF-21 刺激作用的生物活性反应, 揭示了 FGF-21 处理能使分化的脂肪细胞中这 2 种受体磷酸 化, 对前脂肪细胞中这 2 种受体无作用. 与许多生长 因子一样, FGF-21 持续处理脂肪细胞能减少脂肪细 胞中这 2 种受体, 这可能是由于受体内吞和降解作用 而造成的. 同时, FGF-21 处理也能减少这 2 种受体的 前体蛋白. 为了进一步研究此结果, 本研究检测了 FGF-21 持续处理脂肪细胞后这 2 种受体的 mRNA 转 录水平; 与蛋白结果一致, mRNA转录也明显减少(结 果未显示). 这些结果表明, 配体持续处理脂肪细胞 既可以提高受体的转换速率又可以下调受体的表达. 这些实验数据为阐述 FGF-21 与 FGFR-1 和 FGFR-2
的物理关系提供了证据.

在 3T3-L1 脂肪细胞中, FGF-21 可以上调葡萄糖 吸收作用及 Glut-1 的表达, 而与 Glut-4 无关, 这与 Kharitonenkov 等人 ${ }^{[13]}$ 的结论一致. 研究认为, Glut-1 的上调表达是 FGF-21 促进 3T3-L1 脂肪细胞葡萄糖 吸收作用的机制. 为了研究 FGFR-1 和 FGFR-2 在 FGF-21 诱导的 Glut-1 上调表达中的作用, 本研究针 对这 2 种受体设计了 RNA 干涉实验. 结果表明, 这 2 种受体特异性的 siRNA 减弱了 FGF-21 诱导的 Glut-1 上调表达，揭示了这 2 种受体在 FGF-21 诱导的 Glut-1 上调表达中是必需的. 此实验结果支持了本研 究所提出的论断, 即 FGF-21 通过 FGFR-1 和 FGFR-2
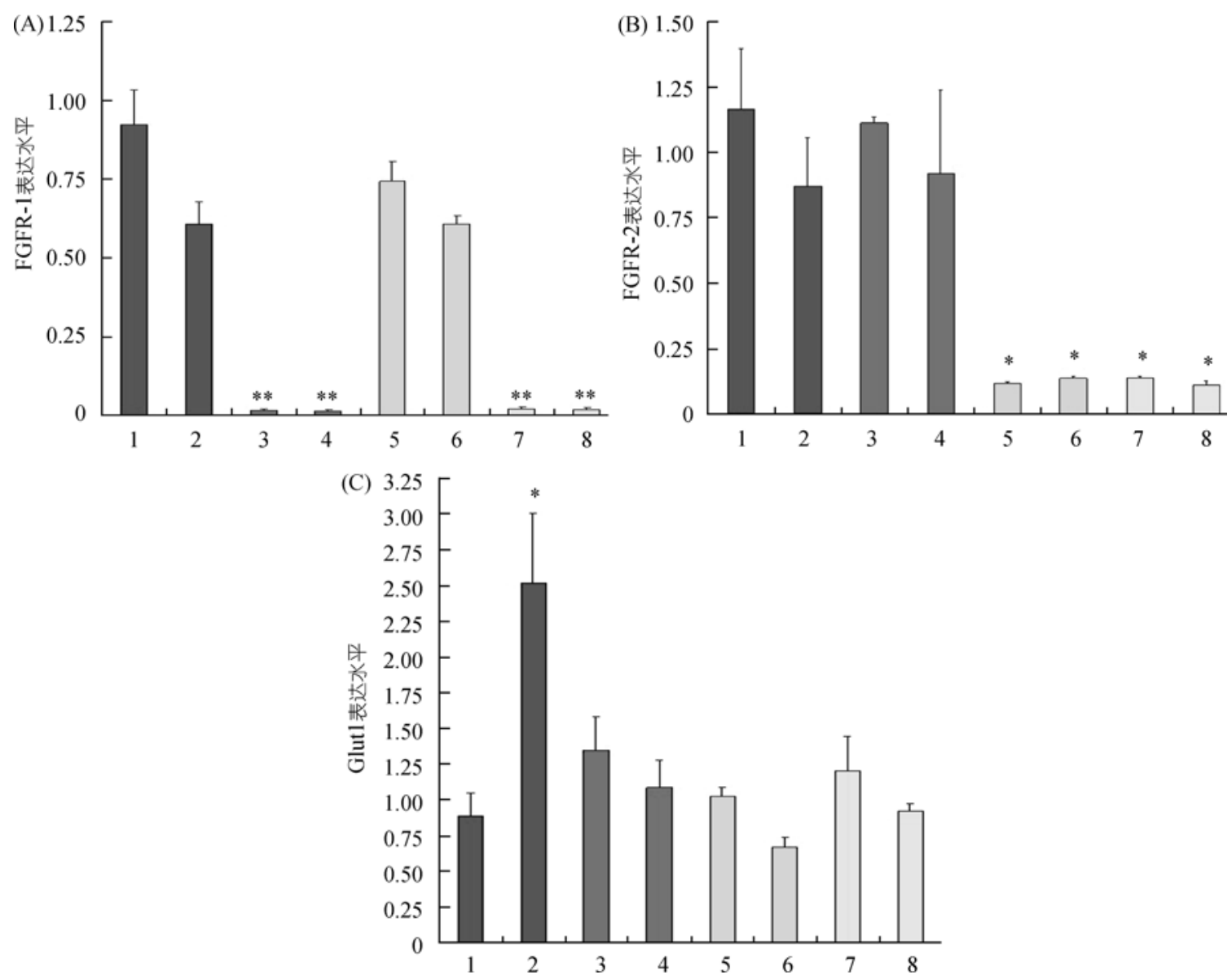

图 6 FGFR-1 和 FGFR-2 基因特异性的 siRNA 对 FGF-21 诱导 Glut1 上调表达的作用

（A）实时荧光定量 PCR 分析基因特异性的 siRNA 对 FGFR-1 表达水平的影响; (B) 实时荧光定量 PCR 分析基因特异性的 siRNA 对 FGFR-2 表达水平的影响; (C) 实时苂光定量 PCR 分析受体表达量的减少对 Glut1 的调控作用. 每个样品至少重复 3 次; 两样本比较的 $t$ 检验, $*$ P 0.05 或 $* * P<0.01$ 差异显著. 1: 不规则 siRNA; 2: 不规则 siRNA, FGF-21 处理 4 h; 3: FGFR-1 siRNA; 4: FGFR-1 siRNA, FGF-21 处理 4 h;

5: FGFR-2 siRNA; 6: FGFR-2 siRNA, FGF-21 处理 4 h; 7: FGFR-1 和 FGFR-2 siRNA; 8: FGFR-1 和 FGFR-2 siRNA, FGF-21 处理 4 h 
介导其上调 Glut-1 表达的作用. 综上, 本研究提出假 设, 即在 3T3-L1 脂肪细胞中 FGF-21 通过 FGFR-1 和 FGFR-2 介导其葡萄糖代谢作用.

这 2 种受体的哪种剪切异构体在 FGF-21 诱导的 葡萄糖的吸收中发挥作用? 为了解决这个问题, 在 3T3-L1 成纤维细胞和脂肪细胞的每个受体中随机挑 取 100 个阳性克隆进行序列分析. 发现 3T3-L1 成纤 维细胞和脂肪细胞中的 FGFR-1 和 FGFR-2 的剪切异 构体均是IIII. 尽管 3T3-L1 细胞中也表达 FGFR-3, 但是在 FGF-21/受体复合物中检测不到 FGFR-3 的存 在, 并且 FGF-21 不能使 FGFR-3 磷酸化(结果未显示), 因此 FGFR-3 不是 FGF-21 的功能受体. 据报道, 在
FGF-19 转基因鼠中 FGF-19 也能促进糖代谢和能量 消耗. 由于 FGF-21 和 FGF-19 在序列及与糖代谢有关 的生理功能上的相似性, 最初研究认为 FGFR-4 是 FGF-21 的功能受体 ${ }^{[10,12,18]}$. 但是在 3T3-L1 细胞中根 本不表达 FGFR-4, 本研究通过免疫印迹、各种 RT-PCR 及实时苂光定量 PCR 证实了这一结论(结果 未显示). 由于在 3T3-L1 细胞中不表达 FGFR-1 和 FGFR-2 其他类型的剪切异构体, 因此FGF-21 可能是 通过 FGFR-1IIIc 和 FGFR-2IIIc 调控糖代谢. Hart 等 人 ${ }^{[19]}$ 检测了胰腺中 FGFR-1 和 FGFR-2 剪切异构体的 生理功能.他们发现, 减弱 FGFR-1IIIc 信号的转基因 鼠最终发展成糖尿病, 而减弱 FGFR-2IIIb 信号的转

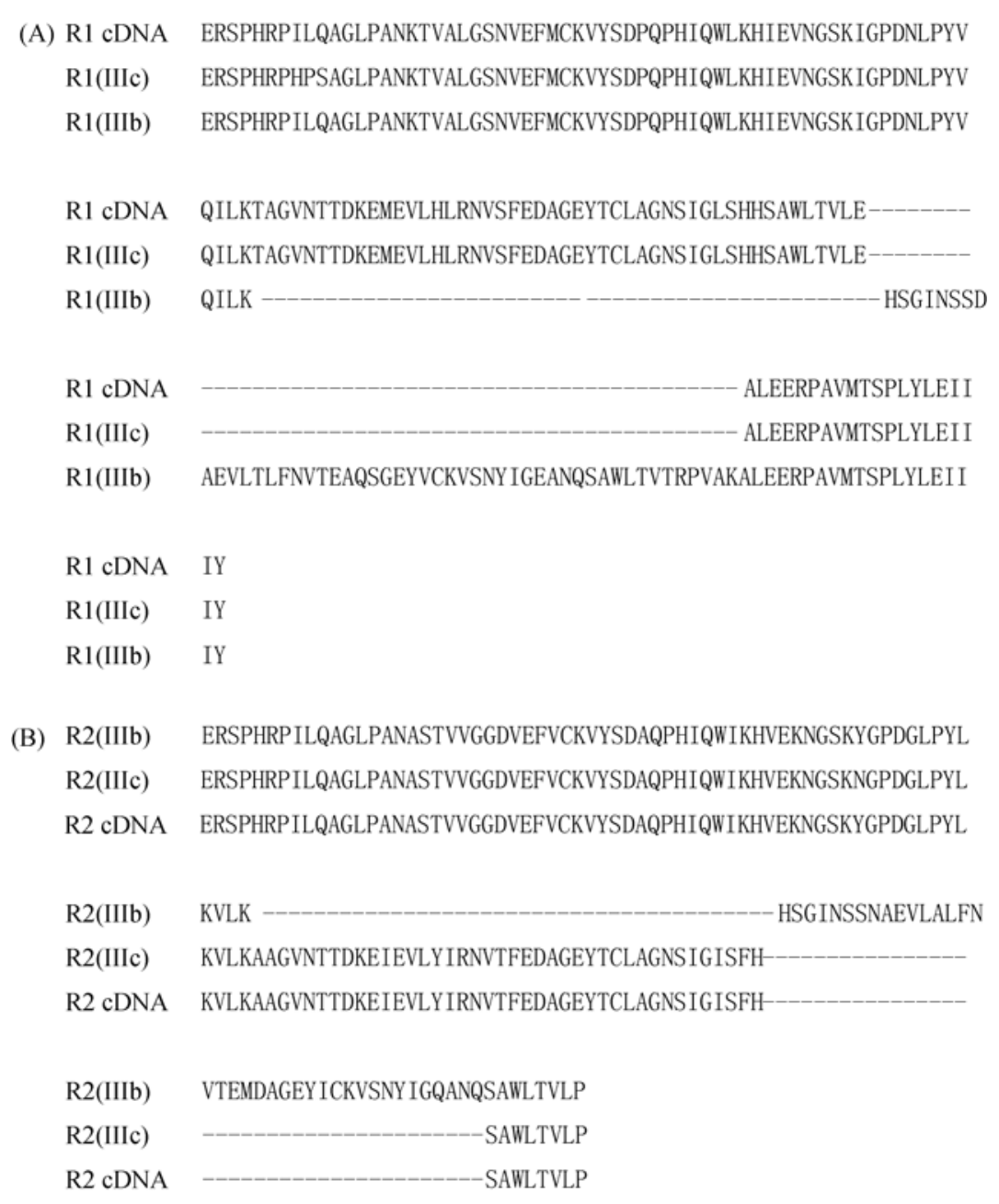

图 7 FGFR-1 和 FGFR-2 的 IgIIIc 序列分别与已发表的鼠源相应剪切异构体的序列比较

(A) FGFR-1 的 IgIII cDNA 序列分别与 NCBI 上已发表的鼠源 FGFR-1 的 IgIIIb 和 IgIIIc 剪切异构体进行比较; (B) FGFR-2 的 IgIII cDNA 序列分别与 NCBI 上已发表的鼠源 FGFR-2 的 IgIIIb 和 IgIIIc 剪切异构体进行比较 
基因鼠不会发展成糖尿病. 本研究所获得的数据与 Hart 等人的发现一致, FGFR-1IIIc 和 FGFR-2IIIc 参与 脂肪细胞的糖代谢.

本研究表明, 在 3T3-L1 成纤维细胞和分化的 3T3-L1 脂肪细胞中表达相同的 FGFR-1 和 FGFR-2 的 剪切异构体，但是这些受体仅在分化的脂肪细胞中 发挥作用. 一种可能性是在此 2 种细胞中这些受体具 有不同的翻译后修饰. 与 3T3-L1 成纤维细胞相比, 3T3-L1 脂肪细胞中的 FGFR-2 有微小的迁移(结果未 显示), 其原因还不清楚. 另一种可能是在此 2 种细胞 中这些受体具有不同的转换率.一方面, 在蛋白水平 上，发现 3T3-L1 脂肪细胞中表达大量 FGFR-1 和 FGFR-2 的前体蛋白, 而在 3T3-L1 成纤维细胞中很少. 另一方面，在 RNA 水平上, 3T3-L1 成纤维细胞分化 后 FGFR-1 和 FGFR-2 的表达增加, 但是成熟蛋白没
有明显变化(结果未显示). 目前, 还不清楚蛋白转换 率是如何影响受体功能的. 第 3 种可能是协同因子或 协同受体参与受体的结构和活性, 这些因子可能仅 存在于分化的脂肪细胞中. 大多数 FGF 家族成员发 挥作用时需要 HLGAGs 的参与. 尽管 FGF-21 发挥作 用时不需要肝素的参与, 但是在 FGF-21 与其受体相 互作用时可能需要肝素替代分子的参与, 而此分子 仅在分化的脂肪细胞中表达. Rusnati 等人 ${ }^{[20]}$ 报道, 细 胞相关的神经节苷脂能与 FGF-2 的 C 末端结合, 并作 为 FGF-2 的协同受体发挥作用. FGF 结合蛋白 1(FGF-BP1)能与 FGF-1 和 FGF-2 特异性地结合, 并 作为分子伴侣发挥作用. 据报道, FGF-BP1 可以增强 FGF-1 和 FGFR-2 依赖性的 NIH-3T3 细胞增殖 ${ }^{[21]}$. 3T3-L1 成纤维细胞分化后, 协同受体或分子伴侣才 能表达. 究竟是哪种机制? 还有待进一步研究.

\section{参考文献}

1 Powers C J, McLeskey S W, Wellstein A. Fibroblast growth factors, their receptors and signaling. Endocrine-Related Cancer, 2000, 7: 165197

2 Eswarakumar V P, Lax I, Schlessinger J. Cellular signaling by fibroblast growth factor receptors. Cytokine Growth Factor Rev, 2005, 16: $139-149$

3 Yamashita T, Yoshioka M, Itoh N. Identification of a novel fibroblast growth factor, FGF-23, preferentially expressed in the ventrolateral thalamic nucleus of the brain. Biochem Biophys Res Commol/Lun, 2000, 277: 494-498

4 Yeh B K, Igarashi M, Eliseenkova A V, et al. Structural basis by which alternative splicing confers specificity in fibroblast growth factor receptors. Proc Natl Acad Sci USA, 2003, 100: 2266-2271

5 Olsen S K, Ibrahimi O A, Raucci A, et al. Insights into the molecular basis for fibroblast growth factor receptor autoinhibition and ligand-binding promiscuity. Proc Natl Acad Sci USA, 2004, 101: 935-940

6 Pellegrini L, Burke D F, von Delft F, et al. Crystal structure of fibroblast growth factor receptor ectodomain bound to ligand and heparin. Nature, 2000, 407: 1029-1034

7 Plotnikov A N, Schlessinger J, Hubbard S R, et al. Structural basis for FGF receptor dimerization and activation. Cell, 1999, 98: 641—650

8 Bellot F, CrumLey G, Kaplow J M, et al. Ligand-induced transphosphorylation between different FGF receptors. EMBO, 1991, 10: 28492854

9 Schlessinger J, Plotnikov A N, Ibrahimi O A, et al. Crystal structure of a ternary FGF-FGFR-heparin complex reveals a dual role for heparin in FGFR binding and dimerization. Molecular cell, 2000, 6: 743-750

10 Xie M H, Holcomb I, Deuel B, et al. FGF-19, a novel fibroblast growth factor with unique specificity for FGFR4. Cytokine, 1999, 11: 729-735

11 Ezzat S, Asa S L. FGF receptor signaling at the crossroads of endocrine homeostasis and tumorigenesis. Horm Metab Res, 2005, 37: 355-360

12 Tomlinson E, Fu L, John L, et al. Transgenic mice expressing human fibroblast growth factor-19 display increased metabolic rate and decreased adiposity. Endocrinology, 2002, 143: 1741-1747

13 Kharitonenkov A, Shiyanova T L, Li D S, et al. FGF-21 as a novel metabolic regulator. J Clin Invest, 2005, 115: 1627-1635

14 Nishimura T, Nakatake Y, Konishi M, et al. Identification of a novel FGF, FGF-21, preferentially expressed in the liver. Biochim Biophys Acta, 2000, 1492: 203-206 
15 任桂萍, 李璐, 孙国鹏, 等. 成纤维细胞生长因子(FGF)受体-2 参与 FGF-21 介导的糖代谢活性. 生物化学与生物物理进展, 2009, 36: $165-174$

16 姜媛媛, 刘铭瑶, 任桂萍, 等. 鼠源成纤维细胞生长因子-21 对脂肪细胞糖代谢的作用. 生物化学与生物物理进展, 2009, 36: 157一 164

17 王文飞, 任桂萍, 侯玉婷, 等. 3T3-L1 脂肪细胞膜 FGF-21 结合蛋白的初步鉴定. 中国生物化学与分子生物学报, 2008, 24: 667一673

18 Rusnati M, Tanghetti E, Urbinati C, et al. Interaction of fibroblast growth factor-2 (FGF-2) with free gangliosides: biochemical characterization and biological consequences in endothelial cell cultures. Mol Biol Cell, 1999, 10: 313-327

19 Hart A W, Baeza N, Apelqvist A, et al. Attenuation of FGF signalling in mouse beta-cells leads to diabetes. Nature, 2000, 408: 864-868

20 Rusnati M, Urbinati C, Tanghetti E, et al. Cell membrane GM1 ganglioside is a functional coreceptor for fibroblast growth factor 2. Proc Natl Acad Sci USA, 2002, 99: 4367-4372

21 Tassi E, Al-Attar A, Aigner A, et al. Enhancement of fibroblast growth factor (FGF) activity by an FGF-binding protein. J Biol Chem, 2001, 276: $40247-40253$ 